\title{
Novel approach with intratracheal administration of microgelatin hydrogel microspheres incorporating basic fibroblast growth factor for rescue of rats with monocrotaline-induced pulmonary hypertension
}

Keiichi Hirose, MD, PhD, ${ }^{a}$ Akira Marui, MD, PhD, ${ }^{\mathrm{b}}$ Yoshio Arai, MD, PhD, ${ }^{\mathrm{b}}$ Toshihiro Kushibiki, PhD, ${ }^{\mathrm{c}}$ Yu Kimura, MEng, Hisashi Sakaguchi, MD, ${ }^{\mathrm{b}}$ Huang Yuang, MD, ${ }^{\mathrm{b}}$ B. I. R. Shyamal Chandra, MD, ${ }^{\mathrm{b}}$ Tadashi Ikeda, MD, PhD, ${ }^{\mathrm{b}}$ Shigeru Amano, MD, $\mathrm{PhD},{ }^{\mathrm{d}}$ Yasuhiko Tabata, PhD, DMSc, Dpharm, ${ }^{\mathrm{c}}$ and Masashi Komeda, MD, $\mathrm{PhD}^{\mathrm{b}}$

Objectives: Pulmonary hypertension is a life-threatening disease, and alternative strategies are essential for patients with critical pulmonary hypertension. We developed a new procedure using microgelatin hydrogel microspheres incorporating basic fibroblast growth factor (mGHMs/bFGF) for intratracheal administration and evaluated the effect of a single intratracheal administration of $\mathrm{mGHMs} / \mathrm{bFGF}$ on rats with monocrotaline-induced pulmonary hypertension.

\begin{abstract}
Methods: Monocrotaline was injected into 54 rats simultaneously with intratracheal administration of plain mGHMs (vehicle group), bFGF in solution form (free-bFGF group), mGHMs/bFGF (mGHMs/bFGF group), and plain saline with subcutaneous injection of saline instead of monocrotaline (control group, $\mathrm{n}=18$ ). Three weeks after the administration, 48 rats ( $n=12$ from each group) were subjected to hemodynamic and histologic evaluations. Survival was assessed in 6 rats of each group 10 weeks after the intratracheal administration.
\end{abstract}

Results: The mGHMs/bFGF group showed significantly lower right ventricular/left ventricular pressure ratios at 3 weeks than the vehicle and free-bFGF groups $(0.35 \pm 0.04,0.54 \pm 0.11,0.58 \pm 0.21$, and $0.36 \pm 0.05$ for the control, vehicle, free-bFGF, and mGHMs/bFGF groups, respectively; $P<.01)$. Histologically, the mGHMs/ bFGF group had a significantly higher number of vessels (diameter $\geq 50 \mu \mathrm{m})$ than the other groups $(5.3 \pm$ $2.6,4.6 \pm 2.8,7.3 \pm 2.5$, and $18.9 \pm 7.0$ vessels $/ \mathrm{mm}^{2}$, respectively; $\left.P<.01\right)$. Ten weeks after the intratracheal administration, $6(100.0 \%)$ rats had survived in the control group, and $1(16.7 \%)$ survived in the vehicle, $0(0 \%)$ in the free-bFGF, and $5(83.3 \%)$ in the $\mathrm{mGHMs} / \mathrm{bFGF}$ groups $(\mathrm{n}=6$ each).

Conclusions: A single intratracheal administration of $\mathrm{mGHMs} / \mathrm{bFGF}$ increased the number of vessels in the lung and ameliorated survival and hemodynamics in rats with monocrotaline-induced pulmonary hypertension.

Pulmonary hypertension (PH) is a life-threatening disease characterized by progressive pulmonary arterial hypertension and increases in pulmonary vascular resistance that lead to right ventricular (RV) failure. ${ }^{1}$ Recently, survival and quality of life have improved in patients with $\mathrm{PH}$ because of the development of pharmacologic therapies, such as novel types of prostacyclin, ${ }^{2,3}$ endothelin receptor blockers, ${ }^{4,5}$ and phosphodiesterase inhibitors. ${ }^{6,7}$ However, these pharmacologic effects might be transient or not sufficient for the severely damaged pulmonary vessels, such as critical PH. Thus alternative strategies are essential for patients with critical $\mathrm{PH}$.

From the Department of Cardiovascular Surgery, ${ }^{\text {a }}$ Shizuoka Children's Hospital, Shizuoka, Japan; the Departments of Cardiovascular Surgery ${ }^{\mathrm{b}}$ and Laboratory Science, ${ }^{\text {d }}$ Kyoto University Graduate School of Medicine, Kyoto, Japan; and the Institute for Frontier Medical Sciences, ${ }^{\mathrm{c}}$ Kyoto University, Kyoto, Japan.

Received for publication Sept 30, 2007; revisions received March 28, 2008; accepted for publication May 9, 2008.

Address for reprints: Masashi Komeda, MD, PhD, Department of Cardiovascular Surgery, Kyoto University Graduate School of Medicine, 54 Shogoin-Kawara-cho,

Sakyo-ku, Kyoto, 606-8507, Japan (E-mail: komelab@kuhp.kyoto-u.ac.jp).

J Thorac Cardiovasc Surg 2008; 136:1250-6

$0022-5223 / \$ 34.00$

Copyright (c) 2008 by The American Association for Thoracic Surgery

doi: $10.1016 /$ j.jtcvs. 2008.05 .038
Basic fibroblast growth factor (bFGF) is not only a potent angiogenic mitogen of various kinds of cells but also a cytokine that can stimulate tissue regeneration. ${ }^{8-10}$ We developed a biodegradable hydrogel made from acidic gelatin as the slow-release carrier for bFGF and demonstrated that sustained release of bFGF with biodegradable gelatin hydrogels enhanced angiogenesis and arteriogenesis in various cardiovascular areas. ${ }^{11-14}$ Although the angiogenic and arteriogenic properties of bFGF can ameliorate severe $\mathrm{PH}$, the optimal delivery method to the lung has not been established. In the study presented here, we used newly developed microgelatin hydrogel microspheres (mGHMs) that make intratracheal administration possible.

We used rats with monocrotaline (MCT)-induced $\mathrm{PH}$ to test the hypothesis that a single intratracheal administration of mGHMs incorporating bFGF (mGHMs/bFGF) could enhance both angiogenesis and arteriogenesis in the lung and ameliorate progressive $\mathrm{PH}$.

\section{MATERIALS AND METHODS Preparation of $\mathbf{m G H M s / b F G F}$ \\ Gelatin with an isoelectric point of 4.9 was isolated from bovine bone collagen by using an alkaline process with $\mathrm{Ca}(\mathrm{OH})_{2}$ (Nitta Gelatin Co, Osaka, Japan). Human recombinant bFGF with an isoelectric point of 9.6}




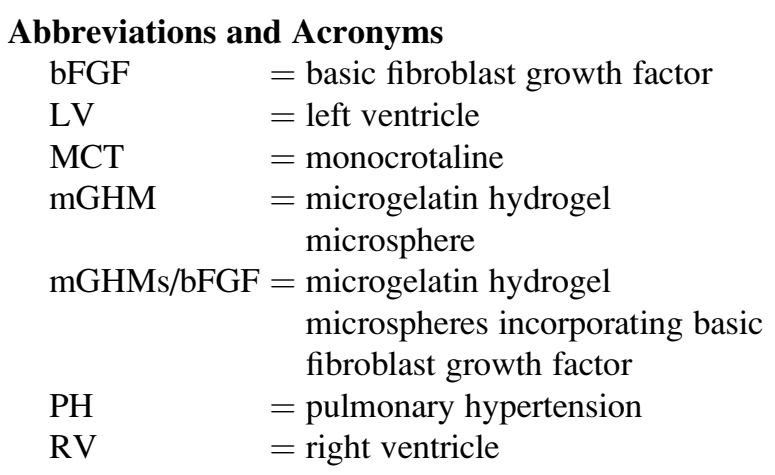

was purchased from Kaken Pharmaceutical Co, Ltd (Tokyo, Japan). mGHMs with a diameter of approximately $10 \mu \mathrm{m}$ were made as previously described ${ }^{15}$ and impregnated with an aqueous solution containing $100 \mu \mathrm{g}$ of bFGF and mGHMs/bFGF. All experimental procedures were conducted under sterile conditions.

\section{Animals}

Male Wistar rats weighing 200 to $220 \mathrm{~g}$ were used in this study. All animals received humane care in compliance with the "Principles of laboratory animal care" formulated by the National Society for Medical Research and the "Guide for the care and use of laboratory animals" prepared by the Institute of Laboratory Animal Resources, National Research Council, and published by the National Academy Press.

\section{Experimental PH Model}

MCT (300 mg, Crotaline; Sigma, St Louis, Mo) was dissolved in $1.8 \mathrm{~mL}$ of $1 \mathrm{~mol} / \mathrm{L} \mathrm{HCl}$, followed by the addition of 3 to $4 \mathrm{~mL}$ of distilled water. ${ }^{16}$ This solution was then adjusted to $\mathrm{pH} 7.4$ with $1 \mathrm{~mol} / \mathrm{L} \mathrm{NaOH}$ solution and topped up to $15 \mathrm{~mL}$ with distilled water. Each rat was anesthetized with an intraperitoneal injection of sodium pentobarbital $(30 \mathrm{mg} / \mathrm{kg})$ and received a single subcutaneous injection of MCT solution $(60 \mathrm{mg} / \mathrm{kg})$ in the neck. After this injection, PH gradually progressed and was completed approximately 3 weeks after the injection, ${ }^{16}$ whereas lung tissues displayed interstitial thickening accompanied by prominent medial hypertrophy of the muscular pulmonary artery and arterioles. ${ }^{17}$

\section{Release Profile of bFGF From mGHMs in Lung Tissue After Intratracheal Administration}

The intrapulmonary release profile of bFGF from mGHMs after intratracheal administration was evaluated in 30 healthy rats. The rats were anesthetized as described above, and mGHMs incorporating iodine 125-labeled $\operatorname{bFGF}(100 \mu \mathrm{g} / 0.1 \mathrm{~mL})$ were injected into the trachea of 15 rats with a pulmonary drug delivery device (Micro Sprayer IA-1B; LMS Co, Ltd, Tokyo, Japan; $\mathrm{mGHMs} / \mathrm{bFGF}$ group, $\mathrm{n}=15$ ). In the other 15 rats, iodine 125-labeled bFGF solution $(100 \mu \mathrm{g} / 0.1 \mathrm{~mL})$ was injected into the trachea with the same device (free-bFGF group, $\mathrm{n}=15$ ). Bilateral pulmonary tissues were obtained 2 hours and $3,7,10$, and 14 days after the intratracheal administration $(n=3$ for each time point). The radioactivity remaining in each lung was measured with a gamma counter (ARC-301B; Aloka, Tokyo, Japan).

\section{Prevention of Progression of PH by mGHMs/bFGF}

Just after the MCT injection, 54 rats were randomized to one of 3 groups $(\mathrm{n}=18$ each) and underwent intratracheal administration of the following solutions: $0.1 \mathrm{~mL}$ of saline containing free mGHMs (vehicle group), 0.1 $\mathrm{mL}$ of saline containing $100 \mu \mathrm{g}$ of bFGF (free-bFGF group), and $0.1 \mathrm{~mL}$ of saline containing mGHMs incorporating $100 \mu \mathrm{g}$ of bFGF (mGHMs/
bFGF group). The remaining 18 rats received a single subcutaneous injection of saline instead of MCT, followed by intratracheal administration of $0.1 \mathrm{~mL}$ of saline (control group). Three weeks after the administration, 12 rats from each group were subjected to hemodynamic and histologic evaluations. Survival was assessed in 6 rats of each group 10 weeks after the intratracheal administration.

\section{Measurement of Hemodynamic Parameters and Assessment of RV Hypertrophy}

Three weeks after the intratracheal administration, rats were anesthetized and mechanically ventilated (fraction of inspired oxygen, 0.21 ; respiratory rate, 60 breaths $/ \mathrm{min}$ ). After the heart was exposed after median sternotomy, systolic pressures of the RV and left ventricle (LV) were measured by means of puncture with a 23-gauge needle. The rats were then killed by means of intravenous administration of a lethal dose of sodium pentobarbital, and the heart and bilateral lungs were thoroughly washed with saline and sampled en bloc under pressurization through an intubation catheter. To assess RV hypertrophy, the RV free wall was dissected from the LV and septum, and they were weighed separately with an analytic scale. Lungs were fixed with $10 \%$ paraformaldehyde solution in PBS solution for histologic examination.

\section{Arterial Blood Gas Analysis}

In conjunction with the hemodynamic studies, an arterial blood sample of approximately $0.2 \mathrm{~mL}$ was obtained from the needle inserted into the LV and subjected to blood gas analysis.

\section{Histopathology}

For the production of paraffin sections, the lung tissue was fixed in formalin for 24 hours at room temperature, dehydrated in ethanol, and embedded in a paraffin block. Sections were cut into $3-\mu \mathrm{m}$-thick specimens and stained with hematoxylin and eosin. Tissue sections were also immunostained with antibodies against von Willebrand factor (an endothelial marker). Normal immunoglobulin fractions were used as a negative control to determine antibody specificity. Five fields were randomly selected in each sample, and the numbers of vessels ( $50 \mu \mathrm{m} \leq$ diameter) and small vessels, capillaries, or both (diameter $<50 \mu \mathrm{m}$ ) per square millimeter were counted manually by 2 pathologists who were unaware of the treatment groups. Medial wall thickness was also assessed as the percentage of medial wall thickness by 2 pathologists using elastica van Gieson stain, as described previously. ${ }^{18}$

\section{Statistical Analysis}

All data was expressed as means \pm standard deviations and as ranges. Differences between groups were assessed by means of analysis of variance, followed by post hoc comparisons with the Bonferroni/Dunn method. Significance of differences in survival data were determined by means of the Kaplan-Meier analysis and compared by using log-rank tests. Statview software (Abacus Concepts, Inc, Berkeley, Calif) was used for all statistical analyses.

\section{RESULTS}

\section{Release Profile of bFGF From mGHMs in the Lung After Intratracheal Administration}

None of the mice died after the intratracheal administration of free-bFGF or mGHMs/bFGF. bFGF was distributed equally in both the mGHMs and mGHMs/bFGF groups in terms of the volume in each lobe of the lung.(Radioactivity remaining in the right upper lobe, right middle lobe, right lower lobe, left upper lobe, and left lower lobe of total lung 2 hours after the intratracheal administration was $10.5 \% \pm 3.9 \%, 11.3 \% \pm 5.5 \%, 24.6 \% \pm 8.4 \%, 43.8 \%$ 


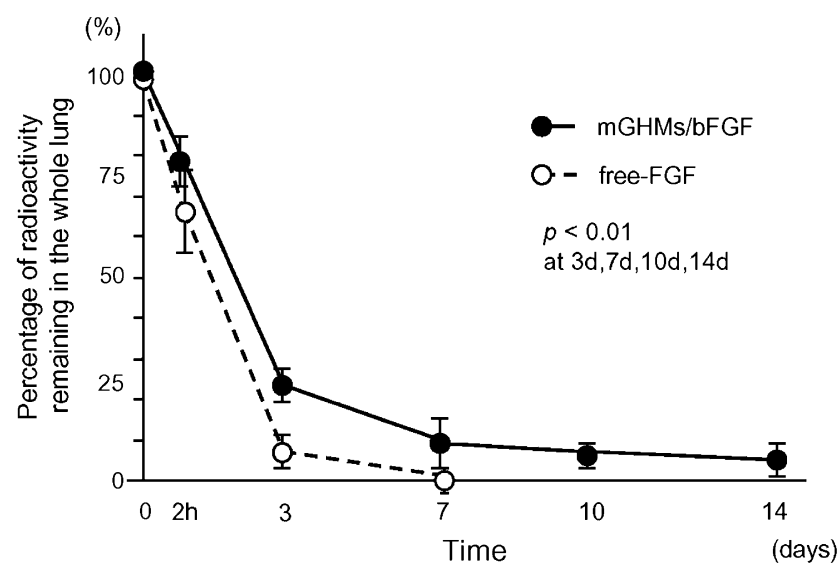

FIGURE 1. Release profile of the radioactivity remaining of basic fibroblast growth factor $(b F G F)$ after intratracheal administration. The $\mathrm{x}$-axis shows the time course and the y-axis shows the percentage of the radioactivity remaining in the lung. $m G H M s / b F G F$ group (filles circles, solid line), Gelatin hydrogel microspheres incorporating iodine 125-labeled bFGF were injected into the trachea by using a pulmonary drug delivery device; free-FGF group (open circles, dotted line): free iodine 125-labeled bFGF solution was injected into the trachea with the same device.

$\pm 17.6 \%$, and $9.9 \% \pm 2.9 \%$, respectively). Radioactivity remaining in the whole lung in the free-bFGF group at 2 hours and 3,7, 10, and 14 days after administration was $67.6 \% \pm 12.6 \%, 7.6 \% \pm 2.2 \%, 2.0 \% \pm 1.2 \%, 0.9 \% \pm$ $0.1 \%$, and $0.1 \% \pm 0.0 \%$, respectively. Corresponding findings for the mGHMs/bFGF group were $71.6 \% \pm 7.2 \%$, $23.1 \% \pm 1.6 \%, 8.3 \% \pm 2.4 \%, 6.1 \% \pm 1.6 \%$, and $5.4 \%$ $\pm 3.1 \%$ (Figure 1). Radioactivity of bFGF remaining in the mGHMs/bFGF group was significantly higher than that in the free-bFGF group at any time point $(P<.05)$. These results suggest that $\mathrm{mGHMs} / \mathrm{bFGF}$ were distributed equally and degraded slowly over 2 weeks after the intratracheal administration. In contrast, the free-bFGF solution was degraded rapidly and almost disappeared in the 3 days after intratracheal administration.

\section{Prevention of Progressive PH by mGHMs/bFGF}

Survival (Figure 2). Survival curves indicated that mGHMs/bFGF significantly improved the survival of rats with progressive PH $(P<.01$; Kaplan-Meier method, logrank test). On day $70,6(100.0 \%)$ rats had survived in the control group, and $1(16.7 \%)$ survived in the vehicle, $0(0 \%)$ in the free-bFGF, and $5(83.3 \%)$ in the mGHMs/ bFGF groups $(\mathrm{n}=6$ each).

Body weights. Body weights 3 weeks after the intratracheal administration of the vehicle and free-bFGF groups were significantly lower than that of the control group. However, there was no significant difference between the control and mGHMs/bFGF groups $(272.9 \pm 4.9,225.0 \pm 16.0,228.3$ \pm 19.4 , and $267.3 \pm 15.6 \mathrm{~g}$ for the control, vehicle, free$\mathrm{bFGF}$, and mGHMs/bFGF groups, respectively; $P<.01$ ).

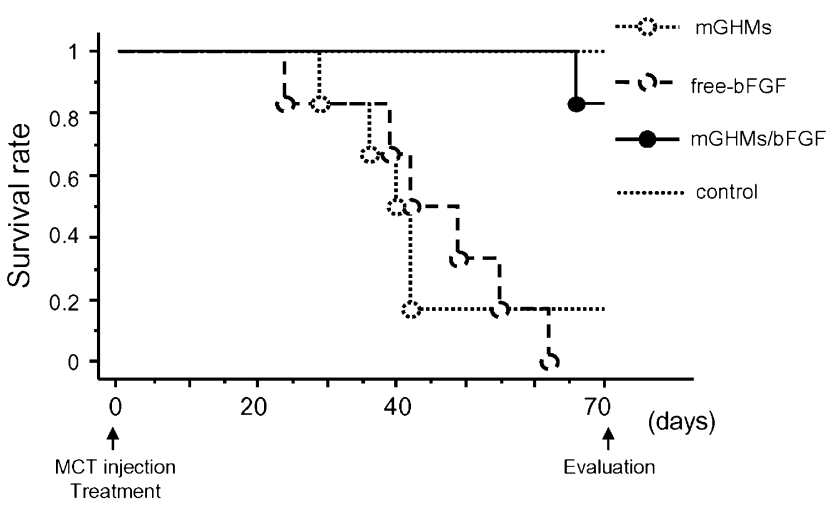

Time

FIGURE 2. Survival curves after a single intratracheal administration into rats with progressive pulmonary hypertension. Survival curves are generated by means of Kaplan-Meier analysis. These results suggest that a single intratracheal administration of microgelatin hydrogels microspheres incorporating basic fibroblast growth factor $(m G H M s / b F G F)$ improved survival of rats with progressive pulmonary hypertension. mGHMs group (open circles, small dotted line), Monocrotaline (MCT)-injected rats (a single subcutaneous injection of monocrotaline) treated with intratracheal administration of plain mGHMs; free-FGF group (open circles, large dotted line), MCTinjected rats treated with intratracheal administration of free-bFGF; $m G H M s / b F G F$ group (closed circles, solid line), MCT-injected rats treated with intratracheal administration of $\mathrm{mGHMs} / \mathrm{bFGF}$ (sustained-release bFGF); control group, healthy rats treated with intratracheal administration of $0.1 \mathrm{~mL}$ of saline (no circles, thin dotted line).

RV pressure. RV/LV pressure ratios of the vehicle and free-bFGF groups were significantly higher than that of the control group, but there was no significant difference between the control and $\mathrm{mGHMs} / \mathrm{bFGF}$ groups $(0.35 \pm 0.04$, $0.54 \pm 0.11,0.58 \pm 0.21$, and $0.36 \pm 0.05 ; P<.01$ for the control, vehicle, free-bFGF, and mGHMs/bFGF groups, respectively; Figure 3, A).

RV hypertrophy. RV/LV plus septum weight ratios of the vehicle and free-bFGF groups were significantly higher than of the control group. However, there was no significant difference between the control group and the $\mathrm{mGHMs} / \mathrm{bFGF}$ group $(0.27 \pm 0.04,0.48 \pm 0.10,0.50 \pm 0.04$, and $0.27 \pm$ $0.15 ; P<.01$ for the control, vehicle, free-bFGF, and mGHMs/bFGF groups, respectively; Figure 3, $B$ ).

Arterial gas analysis. Partial pressure of oxygen in arterial blood of the vehicle and free-bFGF groups was significantly lower than that seen in the control group. However, there was no significant difference between the control group and the mGHMs/bFGF group $(82.2 \pm 3.6,63.8 \pm 5.5$, $67.1 \pm 12.3$, and $80.6 \pm 6.1 \mathrm{~mm} \mathrm{Hg}$ for the control, vehicle, free-bFGF, and $\mathrm{mGHMs} / \mathrm{bFGF}$ groups, respectively; $P<.05)$.

Histology. No bleeding or hematoma was observed after intratracheal administration of mGHMs or mGHMs/bFGF. 

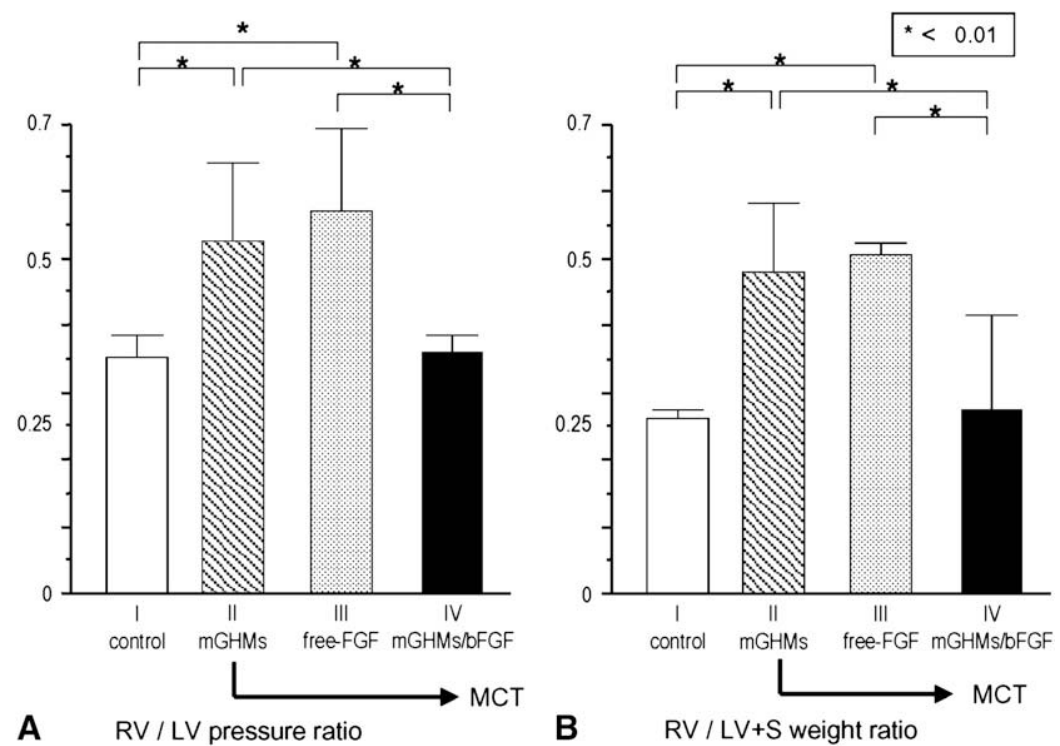

FIGURE 3. Right ventricular $(R V) /$ left ventricular $(L V)$ pressure ratio and $\mathrm{RV} / \mathrm{LV}$ plus septum $(L V+S)$ weight ratio. Panel A shows the RV/LV pressure ratio for the evaluation of pulmonary hypertension 3 weeks after the treatment. Panel B shows the RV/LV+S weight ratio for the evaluation of RV hypertrophy. Control group, Healthy rats treated with intratracheal administration of $0.1 \mathrm{~mL}$ of saline; vehicle group, monocrotaline (MCT)-injected rats (receiving a single subcutaneous injection of monocrotaline) treated with intratracheal administration of plain microgelatin hydrogel microspheres (mGHMs); free-FGF group, MCT-injected rats treated with intratracheal administration of free basic fibroblast growth factor (bFGF); $m G H M s / b F G F$ group, MCT-injected rats treated with intratracheal administration of microgelatin hydrogel microspheres incorporating basic fibroblast growth factor (mGHMs/bFGF; sustained-release bFGF).

Medial wall thickness 3 weeks after treatment in the vehicle and free-bFGF groups was significantly higher than that in the control group and lower, although not significantly lower, in the mGHMs/bFGF group than that in the vehicle and free-bFGF groups $(15.9 \% \pm 1.8 \%, 23.4 \% \pm 3.3 \%$, $22.7 \% \pm 4.6 \%$, and $19.2 \% \pm 3.1 \%$ for the control, vehicle, free-bFGF, and mGHMs/bFGF groups, respectively).

Histologic sections of the lung tissue stained with von Willebrand factor confirmed the increase in the number of vessels and capillaries (Figure 4). The number of vessels (diameter $\geq 50 \mu \mathrm{m}$ ) per square millimeter in the mGHMs/ bFGF group was significantly higher than that in the other 3 groups $(5.3 \pm 2.4,4.1 \pm 2.1,7.3 \pm 2.8$, and $18.8 \pm 7.1$ vessels $/ \mathrm{mm}^{2}$ for the control, vehicle, free-bFGF, and mGHMs/bFGF groups, respectively; $P<.01$; Figure $5, A$ ). The number of small vessels, capillaries, or both (diameter $<50 \mu \mathrm{m}$ ) per square millimeter in the mGHMs/bFGF group was also significantly higher than that in the other 3 groups $(65.5 \pm 16.6,50.5 \pm 12.1,87.7 \pm 17.5$, and $161.0 \pm 43.3$ capillaries $/ \mathrm{mm}^{2}$ for the control, vehicle, free-bFGF, and mGHMs/bFGF groups, respectively; $P<.01$; Figure $5, B$ ). These results indicate that a significant increase in the number of pulmonary vessels and vessel growth was achieved after a single intratracheal administration of $\mathrm{mGHMs} / \mathrm{bFGF}$.

\section{DISCUSSION}

For the study presented here, we newly developed mGHMs that made intratracheal sustained release of bFGF feasible. We were able to demonstrate that a single intratracheal administration of mGHMs/bFGF led to an increase in the number of capillaries and vessels in the lung and attenuated $\mathrm{PH}$ and $\mathrm{RV}$ hypertrophy in rats with progressive $\mathrm{PH}$. Taken together, these findings suggest that a single intratracheal administration of mGHMs/bFGF was effective for the prevention of progressive $\mathrm{PH}$ in rats after MCT injection. To the best of our knowledge, ours is the first study to use an intratracheal sustained release system of angiogenic growth factors for the prevention of progression of $\mathrm{PH}$.

Although bFGF is one of the most potent mitogen-regulating proteins that induces angiogenesis and arteriogenesis among growth factors, the biologic half-life of bFGF in its free form is very short. To promote angiogenesis and arteriogenesis in ischemic tissue with an angiogenic growth factor requires a certain amount of time, whereas maintenance of a significant tissue concentration of bFGF is crucial. In fact, treatment with free-bFGF could not induce sufficient angiogenesis in an ovine tracheal autotransplantation model. ${ }^{18}$ This study showed that the biologic effect of bFGF in its free form was limited but that mGHMs/bFGF was effective for angiogenesis, arteriogenesis, or both in vivo. It was further reported that in vivo gene transfection with a growth factor through the pulmonary artery induced angiogenesis in the rat lung. ${ }^{19}$ However, the problem remains whether sustained protein delivery with genetic materials is safe.

Intratracheal administration was used as the method for administering mGHMs/bFGF in our study, and mGHMs/bFGF 


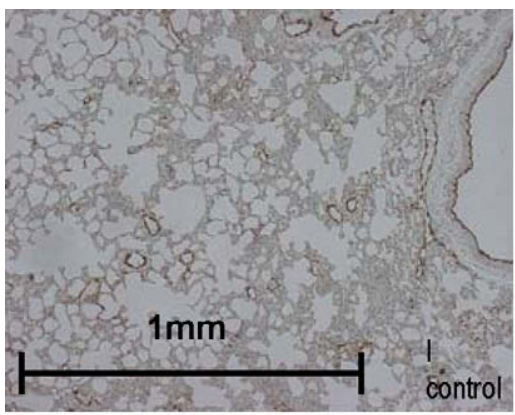

A

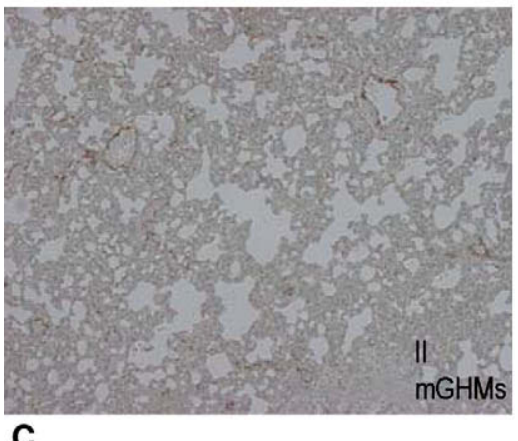

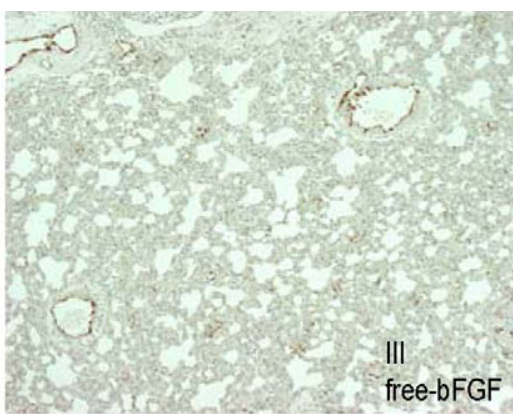

B

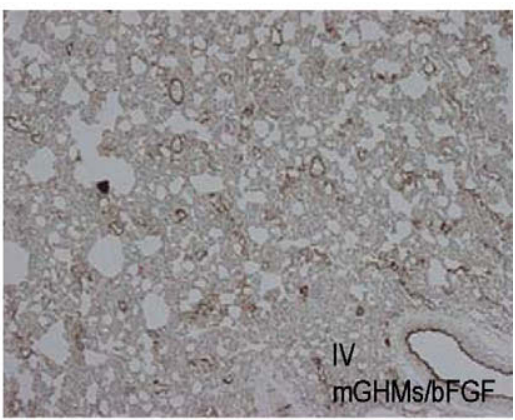

FIGURE 4. Micrographs of the lung tissue. Micrographs of the lung tissue (stained with von Willebrand factor, $100 \times$ magnification) 3 weeks after treatment in rates with progressive pulmonary hypertension. Histologic sections of the lung tissue stained with von Willebrand factor confirmed the increase in the number of vessels and capillaries. Micrograph I (left upper, control group), Healthy rats treated with intratracheal administration of $0.1 \mathrm{~mL}$ saline; Micrograph II (left lower, vehicle group), monocrotaline (MCT)-injected rats (receiving a single subcutaneous injection of monocrotaline) treated with intratracheal administration of plain microgelatin hydrogel microspheres (mGHMs); Micrograph III (right upper, free-FGF group), MCT-injected rats treated with intratracheal administration of free basic fibroblast growth factor (bFGF); Micrograph IV (right lower, $m G H M s / b F G F$ group), MCT-injected rats treated with intratracheal administration of microgelatin hydrogel microspheres incorporating basic fibroblast growth factor (mGHMs/bFGF; sustained-release bFGF).

were degraded slowly in the lung after administration. A single administration through the pulmonary artery might be one of the effective methods for administering mGHMs/ bFGF. However, pulmonary arterial administration of mGHMs/bFGF can cause major pulmonary thrombosis when the diameter of a microsphere is too large, or it can pass through the lung and circulate through the whole body when the diameter is too small. Determination of the diameter of a microsphere is therefore critical for pulmonary arterial administration. On the other hand, although intratracheal administration might cause transient atelectasis in a limited part of the lung, tracheal thrombosis would improve as the mGHMs degrade, and thus systemic invasiveness might be limited. In fact, no complications, such as bleeding or the deterioration of partial pressure of oxygen in arterial blood gas, were found after intratracheal administration of mGHMs/bFGF in our study. Because the serum concentration of bFGF after the intratracheal administration was very low (data not shown), the systemic effect of bFGF, if any, might be minimal. Nor were there any histologic changes in tissues, such as the liver and kidney, after a single intratracheal administration of mGHMs/bFGF in the preliminary study. We therefore believe that intratracheal administration of mGHMs/bFGF for PH can be used safely in the clinical setting. The amount of bFGF obtained in the mGHMs was determined based on the results of other studies of angiogenesis with a single administration of mGHMs/ bFGF. ${ }^{11-14}$

It is tempting to speculate on the underlying mechanisms as to why mGHMs/bFGF were effective for the rats with MCT-induced PH in our study. MCT has been widely used to establish an animal model of PH and reportedly induces lung reactions, such as interstitial edema, inflammation, hemorrhage, and fibrosis. ${ }^{20}$ Subcutaneous injection of MCT thus reduces the pulmonary vascular bed, leading to an increase in pulmonary arterial blood pressure and severe pressure overload-induced RV hypertrophy. A single intratracheal administration of mGHMs/bFGF increased the number of pulmonary vessels as a result of angiogenesis, arteriogenesis, or both in the lung. We assume that these effects of mGHMs/bFGF in the severely injured pulmonary vessels after MCT injection enhance the total pulmonary vascular bed and reduce pulmonary arterial pressure. In addition, the results of the arterial gas analysis indicate that no direct arteriovenous connections in the lung were formed after the single intratracheal administration of $\mathrm{mGHMs} / \mathrm{bFGF}$. For clarification of the details of this mechanism, however, further investigation will be necessary. 

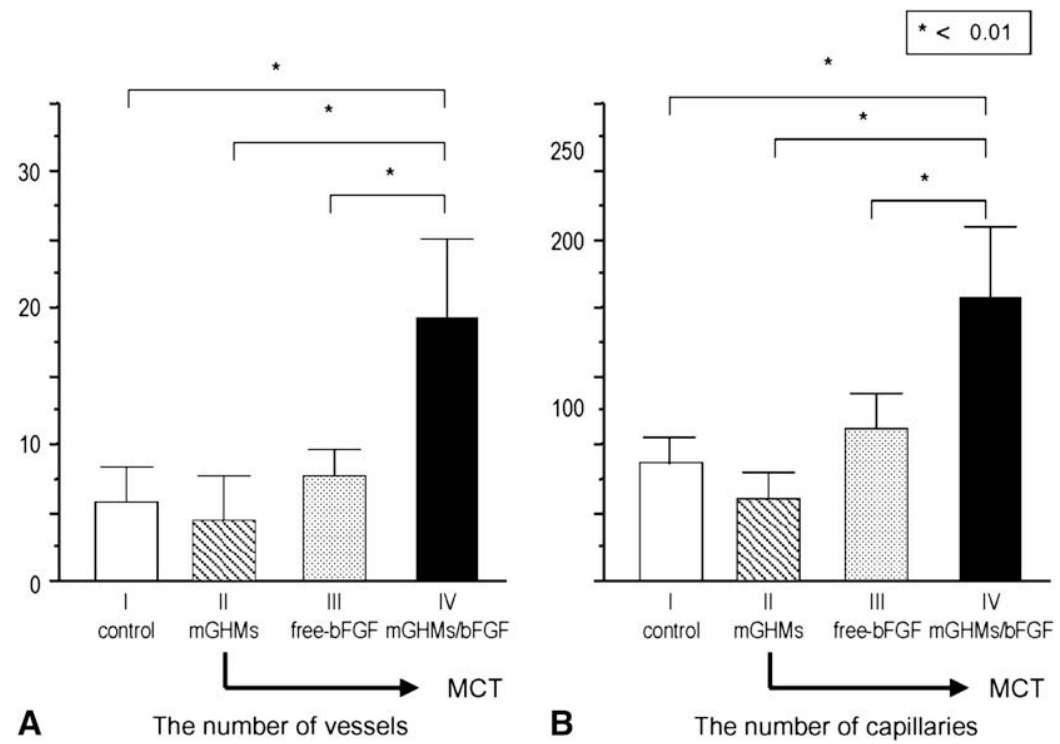

FIGURE 5. The number of vessels and capillaries. The left panel (A) shows the number of vessels (diameter $>50 \mu \mathrm{m}$ ) per square millimeter, and the right panel (B) shows the number of small vessels, capillaries, or both (diameter $<50 \mu \mathrm{m}$ ) per square millimeter 3 weeks after treatment in rats with progressive pulmonary hypertension. Control group, Healthy rats treated with intratracheal administration of $0.1 \mathrm{~mL}$ saline; vehicle group, monocrotaline (MCT)-injected rats (receiving a single subcutaneous injection of monocrotaline) treated with intratracheal administration of plain microgelatin hydrogel microspheres (mGHMs); free-FGF group, MCT-injected rats treated with intratracheal administration of free basic fibroblast growth factor (bFGF); $m G H M s / b F G F$ group, MCT-injected rats treated with intratracheal administration of microgelatin hydrogel microspheres incorporating basic fibroblast growth factor (mGHMs/ bFGF; sustained-release bFGF).

We did not evaluate the effects of intratracheal administration of mGHMs/bFGF in normal murine lung. However, we reported that subcutaneous injection of $\mathrm{mGHMs} / \mathrm{bFGF}$ in healthy animals enhanced vascularization and tissue granulation. ${ }^{21}$ Based on these findings, we speculate that intratracheal administration of mGHMs/bFGF can also enhance neovascularization in normal animal lung tissue. However, further investigation will be necessary to prove this.

The main limitation of this experimental study is that we used MCT-treated rats for our experimental model, which might mean that the $\mathrm{PH}$ was more progressive than that seen in human subjects. It was reported that bFGF levels were increased during the development of MCT-induced $\mathrm{PH}$ in rats. ${ }^{22}$ Of course, we believe that the level of bFGF released from mGHMs/bFGF after intratracheal administration in this study is much higher than the level of bFGF released from endothelial cells intrinsically. Additional investigations with other animal $\mathrm{PH}$ models with pathologic features more similar to those of human patients with $\mathrm{PH}$ are necessary before human studies of this method can be initiated.

The second limitation is that this is a "prevention" study. It is very important to evaluate the effect of the intratracheal administration of mGHMs/bFGF in a "treatment" study. We have some plans to investigate "later administration" with "advanced" PH models in other animals in the next series.

Our strategy with mGHMs/bFGF for the prevention from progression of $\mathrm{PH}$ is intended to be combined with cardiac surgery. The therapeutic approach developed by us has the potential to improve lung conditions (or prevent progression of $\mathrm{PH}$ ) before surgical intervention, such as staged operations for Fontan candidates in the clinical setting. Although our intratracheal administration method involved injection of the solution with $\mathrm{mGHMs} / \mathrm{bFGF}$ into the trachea with a pulmonary drug delivery device, it might be necessary to develop mGHMs further so that for clinical use they can be inhaled into the trachea in aerosol form.

In conclusion, a single intratracheal administration of mGHMs/bFGF increased the number of vessels in the lung and improved hemodynamics and survival with potential improvement of gas exchange in rats with MCT-induced $\mathrm{PH}$. These findings indicate an important role for mGHMs/bFGF in angiogenesis, arteriogenesis, or both in the lung and suggest that this therapeutic approach might provide a novel treatment strategy for patients with primary or secondary PH because this noninvasive treatment is essentially topical, with negligible systemic effects.

We thank Dr Masaaki Imamura for help with the evaluations and Ms Mari Ishii and Maiko Yamamoto for their secretarial assistance.

\section{References}

1. D'Alonzo GE, Barst RJ, Ayres SM, Bergofsky EH, Brubdage BH, Detre KM, et al. Survival in patients with primary pulmonary hypertension. Results from a national prospective registry. Ann Intern Med. 1991;115:343-9.

2. Olschewski H, Simonneau G, Galie N, Higenbottam T, Naeije R, Rubin LJ, et al. Inhaled iloprost for severe pulmonary hypertension. $N$ Engl J Med. 2002;347: 322-9. 
3. Simonneau G, Barst RJ, Galie N, Naeije R, Rich S, Bourge RC, et al. Continuous subcutaneous infusion of treprostinil, a prostacyclin analogue, in patients with pulmonary hypertension: a randomized, double-blind, placebo-controlled trial. Am J Respir Crit Care Med. 2002;165:800-4.

4. Rubin LJ, Badesch DB, Barst RJ, Galie N, Black CM, Keogh A, et al. Bosentan therapy for pulmonary arterial hypertension. N Engl J Med. 2002;346:896-903.

5. Rich S, McLaughlin VV. Endothelin receptor blockers in cardiovascular disease. Circulation. 2003;108:2184-39.

6. Ghofrani HA, Rose F, Schermuly RT, Olschwski H, Wiedemann R, Kreckel A, et al. Oral sildenafil as long-term adjunct therapy to inhaled iloprost in severe pulmonary hypertension. J Am Coll Cardiol. 2003;42:158-64.

7. Schermuly RT, Kreisselmeier KP, Ghofrani HA, Yilmaz H, Butrous G, Ermert L, et al. Chronic sildenafil treatment inhibits monocrotaline-induced pulmonary hypertension in rats. Am J Respir Crit Care Med. 2004;169:39-45.

8. Rafkin DB, Moscatelli D. Recent developments in the cell biology of basic fibroblast growth factor. J Cell Biol. 1989;109:1-6.

9. Yanagisawa-Miwa A, Uchida Y, Nakamura F, Tomaru T, Kido H, Kamijo T, et al. Salvage of infarcted myocardium by angiogenic action of basic fibroblast growth factor. Science. 1992;257:1401-3.

10. Schliephake H, Newkam FW, Lohr A, Hutmacher D. The use of basic fibroblast growth factor (bFGF) for enhancement of bone ingrowth into pyrolized bovine bone. Int J Oral Maxillofac Surg. 1995;24:181-6.

11. Sakakibara Y, Nishimura K, Tambara K, Yamamoto M, Tabata Y, LuF, et al. Prevascularization with gelatin microspheres containing basic fibroblast growth factor enhances the benefits of cardiomyocyte transplantation. J Thorac Cardiovasc Surg. 2002;124:50-6.

12. Ueyama K, Bing G, Tabata Y, Ozeki M, Doi K, Nishimura K, et al. Development of biologic coronary artery bypass grafting in a rabbit model: revival of a classic concept with modern biotechnology. J Thorac Cardiovasc Surg. 2004;127: $1608-15$.
13. Marui A, Kanematsu A, Yamahara Y, Doi K, Kushibiki T, Yamamoto M, et al. Simultaneous application of basic fibroblast growth factor and hepatocyte growth factor to enhance the blood vessels formation. J Vasc Surg. 2005;41:82-90.

14. Hirose K, Fujita M, Marui A, Arai Y, Sakaguchi H, Huang Y, et al. Combined treatment of sustained-release basic fibroblast growth factor and sarpogrelate enhances collateral blood flow effectively in rabbit hindlimb ischemia. Circ J. 2006; 70:1190-4.

15. Tabata Y. Tissue regeneration based on growth factor release. Tissue Eng. 2003; 9(suppl):S5-15.

16. Hayashi Y, Lalich JJ. Renal and pulmonary alterations induced in rats by a single injection of monocrotaline. Proc Soc Exp Biol Med. 1967;124:392-6.

17. Balasubramaniam V, Le Cras TD, Ivy DD, Grover TR, Kinsella JP, Abman SH. Role of platelet-derived growth factor in vascular remodeling during pulmonary hypertension in the ovine fetus. Am J Physiol Lung Cell Mol Physiol. 2003; 284:L826-33.

18. Behrend M, von Wasielewski R, Klempnauer J. Failure of airway healing in an ovine autotransplantation model that includes basic fibroblast growth factor. J Thorac Cardiovasc Surg. 2002;124:231-40.

19. Ono M, Sawa Y, Matsumoto K, Nakamura T, Kaneda Y, Matsuda H. In vivo gene transfection with hepatocyte growth factor via the pulmonary artery induces angiogenesis in the rat lung. Circulation. 2002;106:I264-9.

20. Ghodsi F, Will JA. Changes in pulmonary structure and function induced by monocrotaline intoxication. Am J Physiol Heart Circ Physiol. 1981;240:H149-55.

21. Tabata Y, Hijikata S, Ikada Y. Enhanced vascularization and tissue granulation by basic fibroblast growth factor impregnated in gelatin hydrogels. J Controlled Release. 1994;31:189-99.

22. Arcot SS, Fagerland JA, Lipke DW, Gillespie MN, Olson JW. Basic fibroblast growth factor alternations during development of monocrotaline-induced pulmonary hypertension in rats. Growth Factors. 1995;12:121-30. 